\title{
Conflict between parents over care
}

\author{
Alasdair I. Houston ${ }^{1}$, Tamás Székely ${ }^{2}$ and John M. McNamara ${ }^{3}$ \\ ${ }^{1}$ Centre for Behavioural Biology, School of Biological Sciences, University of Bristol, Bristol, UK, BS8 1 UG \\ ${ }^{2}$ Department of Biology and Biochemistry, University of Bath, Bath, UK, BA2 7AY \\ ${ }^{3}$ Centre for Behavioural Biology, Department of Mathematics, University of Bristol, Bristol, UK, BS8 1TW
}

\begin{abstract}
Conflict between parents over care of young arises when the young benefit from the effort of both parents, but each parent suffers a reduction in future reproductive success as a consequence of its own effort. Here, we review existing models and argue that they fail to capture many important components of parental conflict. For example, we lack adequate models of how a parent should compensate for a reduction in the effort of its mate. These models should incorporate the process by which decisions are reached. Recent theory suggests that a parent benefits by handicapping itself, and more experimental and theoretical work on this topic could be fruitful. We also need more theoretical work on attractiveness that incorporates consistent interactions between males and females.
\end{abstract}

There are many forms of parental care and many ways in which care can be costly to a parent, in that it reduces their reproductive success in other breeding attempts $[1,2]$. Parental care involves a high level of energy expenditure, which might reduce the condition of the parent at the end of the breeding season. This can reduce the future survival of the care-provider and, hence, its probability of breeding the following year. A parent that deserts its mate and young has greater opportunities to pair with a new partner within the current breeding season than does a parent that cares. Even if a male remains with his mate and young, it might be possible to increase his chances of copulating with females other than his mate by decreasing his level of care and devoting more time to courting other females [3]. Such matings are known as extra-pair copulations (EPCs).

Although the details vary across species, the success of the young generally depends on the care from both parents, whereas the cost to a parent depends on its own effort. Thus, a conflict of interest emerges [4,5], with each parent preferring the other to do the hard work. Here, we focus on sexual conflict over care, although the parents might also disagree about family size, timing of breeding or the sex ratio of their offspring [6]. For a broader account of sexual conflict, see [7].

The benefit that results from a particular level of care by a parent depends on the behaviour of its partner, whereas the cost of care in terms of lost mating opportunities depends on the behaviour of other members of the population. For example, if a male deserts, his chances of obtaining a new mate will depend on the number of

Corresponding author: Tamás Székely (T.Szekely@bath.ac.uk).

Available online 2 November 2004 females that have also deserted their mate and are hence available. If a male seeks EPCs, then his chances of obtaining them depend on the receptiveness of females in the population and the extent to which males allocate their time to guarding their mates from other males as opposed to caring for their young or seeking EPCs [8]. These complex interactions make it difficult to use verbal arguments to predict the level of care that will evolve. Theory attempts to predict the outcome of evolution by constructing mathematical models that expose the logic of parental conflict. Here, we consider phenotypic models (Box 1) and discuss the necessary features of an adequate theoretical framework.

There is a gap between theory and experimental tests of the predictions of models, as models that are tested experimentally often lack biological features that are crucial in determining the observed behaviour. We consider three areas in which further theoretical work should be integrated with empirical studies: (i) the extent to which a parent will compensate for a low effort of its mate; (ii) whether an individual can manipulate its mate by handicapping itself so that the mate increases its effort; and (iii) how the attractiveness of a parent influences the conflict over parental care.

\section{Theoretical framework}

Models present a simplified view of the world while trying to capture some of its essential features. We would argue, however, that there has been a tendency to oversimplify. Here, we outline features that can play a key role in determining predictions but that are not always included in models.

\section{Consistency}

When we try to establish the outcome of selection, we cannot consider a focal mated pair of animals in isolation from the rest of the population. In a consistent model, the behaviour of any individual is the best given the behaviour of all other individuals. In other words, it is necessary to adopt a holistic analysis of the population. To illustrate this, suppose that one member of the pair deserts to try to obtain another mate. Its probability of remating depends on the number of single males and females, which depends on the desertion behaviour of other population members. In a consistent model, the behaviour of the focal male and female must represent the behaviour within the whole population. This means that the remating probability cannot be given any value; its value is determined by the behaviour of the focal pair. A consistent model corresponds 


\section{Box 1. Phenotypic models}

Genetic models can be used to follow the way in which a trait changes over time under natural selection. If the environment does not change over time then traits might also stop changing (i.e. evolution reaches a stable endpoint). In trying to understand animal behaviour, a reasonable initial approach is to see whether observed behaviour can be explained as a stable endpoint. The advantage of considering stable endpoints rather than the whole evolutionary trajectory is that these endpoints can usually be understood in phenotypic terms $[56,57]$. A phenotypic characterization of an endpoint is based on suitably defined costs and benefits. At an endpoint, population members are maximizing their net benefit. In the case of parental conflict, it is inevitable that costs and benefits depend on the actions of other individuals. In other words, from a phenotypic point of view, the interaction between population members can be seen as a game. In game theoretic terms, an endpoint of the evolutionary process is a trait value that has the following property: if most population members have this trait value, then no individual can obtain a greater net benefit by changing its trait value [58].

To formalize the game theoretic approach, we define a trait value $y$ to be the resident trait value if most population members have this

to a possible state of the world. By contrast, an inconsistent model might contain features that are contradictory and, hence, cannot correspond to a possible state of the world.

Many models of care incorporate consistent remating probabilities [9-14]. However, some are not consistent, including Maynard Smith's influential Model 2 [14]. In this model, males and females decide whether to care or desert. Parents that care do not have the possibility of remating. In one of the evolutionary outcomes predicted by the model, all males desert to remate and all females care. As Webb et al. [15] point out, this is inconsistent with the assumption that deserting males can remate; if all females are caring, a male that deserts will have no-one to mate with.

The above example shows that including the interaction between population members can eliminate solutions that are not possible states of the world. We illustrate this with two further examples. Kokko [16] considers a model in which females are either faithful or solicit EPCs. If a male suspects his mate of being unfaithful, he can reduce his parental effort, which in turn might favour a decrease in faithfulness. This can lead to a situation in which there are, at most, two stable outcomes; in one of them, some females are faithful and both sexes care; in the other, no females are faithful and typically only females care. Alonzo and Warner [17] model the mating and parental behaviour of the Mediterranean wrasse Symphodus ocellatus. They show that analysing the behaviour of sneakers, nesting males and females in isolation from each other fails to provide realistic predictions. When the interactions between these classes of individual are included in the model, there is only one stable outcome. The model provides a good fit to the empirical data in that it correctly predicts that female spawning and male sneaking are concentrated at very successful nests.

In many populations, males gain matings with females that are not their social partners $[8,18,19]$. As a consequence of these EPCs, a male might not be the father of all the offspring of his partner. It seems intuitively obvious value. Let $W(x, y)$ be the net benefit to an individual with trait value $x$ when the resident trait value is $y$. Then, a necessary condition for a trait value $X^{*}$ to be an endpoint is (Eqn I)

$W(x *, x *) \geq W(x, x *)$ for every trait value $x$

[Eqn I]

In the language of game theory, this means that $x^{*}$ is a Nash equilibrium for the game with payoff function $W$. Although this condition is necessary for stability it is not sufficient. One problem is that the inequality in Eqn1 is not strict, so that it is possible for mutant individuals not having the resident trait $x^{*}$ to do as well as individuals that do have this trait value. These mutants might not be eliminated by natural selection and could increase in frequency as a result of drift. Maynard Smith introduced a second condition that eliminated this possibility and called a trait value satisfying both conditions an evolutionarily stable strategy (ESS). Another problem with Eqn1 is that it says nothing about what will happen to a population whose resident trait value is close, but not equal, to $x^{*}$. The condition of continuous stability [59] ensures that such a population will move towards $x^{*}$. In seeking endpoints, game theoretic models should look for solutions that are both ESSs and continuously stable.

that a male that knows he has lost paternity should reduce his parental effort, but it can be argued that this is not the case: if a male has the same paternity, $p$, in all breeding attempts and is unable to obtain EPCs, then his lifetime reproductive success is the number of offspring reared multiplied by $p$. Thus, if the male is unable to influence his paternity, the parental effort that maximizes his lifetime reproductive success is independent of $p$; that is, paternity should not influence parental effort [20]. However, in a consistent model, it is not possible for all males to have reduced paternity unless some males are gaining EPCs $[21,22]$. Although the effect of paternity depends on details such as the options available to a male [20], there are certainly cases in which a consistent model predicts that males that gain EPCs decrease parental effort if paternity is lost (reviewed in [13]).

\section{Behavioural interactions between parents}

If the parental effort of a starling Sturnus vulgaris is decreased experimentally, its partner responds by increasing its level of care [23]. This compensation, which is found in many species of bird (but see [24]), shows that parents respond to each other's behaviour. There is also evidence for compensation in the burying beetle Nicrophorus vespilloides [25], an insect in which both parents care for the young. Further evidence for responding comes from reviewing the frequency of desertion by each sex in a population. For a given level of desertion, there are too few cases in which both parents desert. This shows that if one parent deserts this affects the other parent, because they tend not to desert [26]. In spite of the evidence for behavioural interactions between parents, and its potential implications for patterns of care [27], few models include such interactions. Preliminary work indicates that predictions depend strongly on the process by which decisions are made [28]. For example, if the male decides his level of care before the female and the female responds to the effort of the male, then the outcome can be less care by the male and more by the female than if the pair did not interact [29]. In reality, parental decisions are likely to be the result of a negotiation process involving repeated 
interactions between the male and the female, rather than a single decision by each parent, and models should take this into account $[12,30]$.

\section{Effects of individual differences within a sex}

There is strong evidence that parents respond to one another in deciding their parental effort. This suggests that they are learning about the ability or willingness of their partner to care, and that there are differences of this sort within either sex (e.g. [31]). From a modelling perspective, such differences are required if we are to understand negotiation, otherwise there is nothing to learn and no point in negotiating. In spite of the crucial role of differences within a sex, only a few models of parental effort have incorporated them. Differences within a sex also have implications for the effects of paternity on parental effort. For example, if males that have low paternity also have a low probability of obtaining EPCs, then these males might devote more care to their young than would either their female partner or high paternity males [13].

\section{Should parents compensate?}

Experiments in which a parent is manipulated by attaching a small weight to it or by clipping some of its feathers aim to see whether a reduction in care by the manipulated parent affects the parental effort of its mate. In particular, studies are concerned with whether the mate fully compensates for any reduction in care by the manipulated parent by increasing its own effort (e.g. feeding rate) so that the total effort of both parents remains the same. The procedure reduced the feeding rate of the manipulated parent in some studies [23,24], but the response of the mate of the manipulated parent varied. In some studies, there was no statistically significant effect on the feeding rate of the mate [24,32], but both increases and decreases have been reported [33,34]. The total feeding rate of the manipulated pair was significantly less than that of control pairs in some studies [23,33], but not significantly different in others [32].

What does theory predict about the response of one parent to a change in effort by its partner? We have argued that parents are likely to negotiate their efforts by (perhaps repeatedly) responding to one another. The response of one parent to a change in effort by the other would then depend on the negotiation rule that the first parent is using. One negotiation rule (the best response rule) that has been suggested is for each parent to always adopt the best effort given the current effort of its partner [35]. If a parent followed this rule, it would increase its effort in response to a decrease in effort by its partner, but not by enough to compensate fully $[29,30]$. Although this rule appears plausible, it is not a stable outcome of evolution: suppose that most members of a population adopted this rule, and consider an individual following a mutant strategy with a low level of effort. When this individual cares, its effort is low and its partner partially compensates by a high level of effort. The result is that, although the young are slightly worse off, the mutant individual is better off overall, because of its reduced effort. Thus, by exploiting the willingness of its partner to compensate, a lazy mutant individual will do better than will other population members, and the mutant strategy will invade [30].

To predict the negotiation rules that will evolve, one must regard these, rather than actual efforts, as being heritable. The female rule will evolve so that it is the best negotiation rule given the male rule, and vice versa [30]. McNamara et al. [30] find the pair of negotiation rules that will evolve when individuals differ in quality. Individuals using these rules compensate less strongly than they do under the best response rule when raising their own effort in response to a reduction in the effort of their partner. Although each parent adopts the best negotiation rule given the rule of its partner, the effort of a parent that results from negotiation is not the best given the negotiated effort of its partner. As a result of these negotiated efforts, the young do less well than if the effort of each parent was the best given the effort of the other $[29,30]$. Under some circumstances, negotiated efforts are so low that the young would do better if cared for by a single parent [29].

The above results are based on particular costs and benefits of care, and the assumption that the negotiation phase does not incur costs. We need more realistic models that go beyond this simple account and, although these models have yet to be developed, it can be argued that full compensation will not be predicted. If there is full compensation, then a parent can decrease its own effort without reducing the total effort to the young. Thus, if parents respond only to each other's effort, then undercompensation seems necessary for evolutionary stability. However, parents might also respond to aspects of the condition of their partner [36], and might be expected to do so if an individual can honestly signal its condition. This suggests that a parent can sometimes gain an advantage by temporarily handicapping itself, provided that this handicap can be observed accurately by its partner.

\section{Do parents gain an advantage by handicapping themselves?}

If a female is caring for young on her own then it is typically advantageous for her to have high, rather than low, energy reserves. However, if the male is also caring, his parental effort might depend on the reserves of the female, and it is no longer clear that high reserves are advantageous to her. One possibility is that females need high energy reserves to care successfully for the young unaided. If this is so, then a female that handicaps herself by strategically reducing her reserves is unable to care alone, thus forcing the male to care [37]. In this way, the female does better than if she had maintained high reserves. Under some circumstances, the female can desert, given that the male is forced to care [37]. These intriguing possibilities have yet to be investigated empirically.

Parents might gain an advantage by handicapping themselves in other ways. Smith and Härdling [38] argue that females might lay clutches that are larger than their optimal value if they had no help from a male. If the male did not provide care for this large clutch, and the female adopted her optimal effort, then the breeding attempt would not be successful. This forces the male to care as 
well. In this example the large clutch can be thought of as a self-imposed handicap. Consistent with the argument of Smith and Härdling, after experimental reduction of clutches, male starlings spent less time incubating and instead attracted a second female [39].

By starting to moult while still feeding the young, male pied flycatchers Ficedula hypoleuca reduce their ability to care for those young. There is indirect empirical evidence that this forces their female to work harder [40]. Here, the potential handicap is the reduced feather condition during moult. Of course, birds have to moult at some time; male moult can only be considered as a strategic handicap if its timing is not optimal in the absence of a response by the female to the condition of the male.

\section{Should attractive individuals care more (or less) than non-attractive ones?}

Observations and experiments provide conflicting results about how attractiveness (typically, of the male) relates to care. The 'good parent hypothesis' [41,42] predicts that traits, such as ornaments and song, should signal the ability of a male to provide care. Some studies support the hypothesis by showing positive relationships between sexually selected traits and paternal care [43-45], whereas others do not find any relationship $[46,47]$.

Attractive males might care less for the offspring than do less attractive ones [48,49], a relationship that is consistent with two hypotheses. First, there could be a tradeoff between caring for young and gaining matings with a new female [3], and it might be advantageous for attractive males to increase their chances of such matings by devoting less effort to their young than would unattractive males. Second, females mated to attractive males might increase their parental effort either to improve the chances of retaining the mate or because the young are more valuable (the 'differential allocation hypothesis' $[48,50])$. Separating these two hypotheses is not straightforward because parents appear to monitor the effort of their mate, so a reduction in male care might be driven by differential female allocation, or vice versa. Box 2 outlines the features that are necessary for a selfconsistent model of parental care and attractiveness.

\section{Future directions}

Although considerable attention has been devoted to the issue of parental conflict since the pioneering work of Trivers [4] and Maynard Smith [14], there are still surprisingly many gaps in both theoretical analysis and information about the biology.

- Negotiation over parental care. We need further theoretical work on the response of a parent to the effort of its mate. Models should consider how efforts might change over the course of a breeding attempt. Individuals might respond directly to the behaviour of their mate, or use cues such as offspring demand. Theoretical work should explore these possibilities and experimental work should establish the form of negotiation in particular cases. Negotiation rules can be investigated by manipulation experiments, which should investigate the responses of parents using repeated manipulations of breeding pairs and a range of treatment levels. Experiments should also compare the effects of handicapping with those of mate removals. There are theoretical reasons to believe that, after removal, the response of the remaining parent should not be the same as the response that would be made if the partner were present but not providing care [29]. When the partner is present, the response will be part of a negotiation strategy that aims to avoid being exploited by a lazy partner.

- Self-imposed handicaps. We need a broader understanding of whether organisms gain an advantage by imposing a handicap, such as reduced energy reserves, on themselves.

- Effect of common interests on conflict. The outcome of conflict is likely to depend on whether parents either breed together once before changing partners or pair for life [51]. It might also depend on whether the parents are related

\section{Box 2. Modelling attractiveness and the conflict over effort}

Data show that females find some males more attractive than others [60]. From a theoretical perspective, understanding this preference in terms of selection requires specification of: (i) the benefits of choosing a mate; these could be direct (e.g. care or territory) or indirect (genetic); and (ii) the costs of choosiness. Benefit and cost functions cannot be assumed in advance; they emerge from a self-consistent account of parental effort and attractiveness of both partners. Such an account involves various dependencies, such as between the effort of an individual and its own attractiveness. For example, the future mating opportunities of a male are determined by his attractiveness to females; but how attractive a female should find the male depends on information that she has about his expected parental effort. No model incorporates a self-consistent account of this interaction. Another interaction occurs between partners over effort, which has been modeled. However, the few models that include differences between individuals are not specifically concerned with attractiveness. The differential allocation hypothesis [48] says that an individual should work harder to keep an attractive mate. There have been no formal models of this hypothesis that incorporate both mate retention and parent effort.

Kokko [61] presents an instructive model of parental care and attractiveness. Males differ in quality and each allocates resources to

advertising to females, care and self-maintenance in the way that maximizes fitness. Kokko shows that, when there are diminishing returns from both advertising and care, the level of advertisement is an honest indicator of male care. At evolutionary stability, males that advertise more also care more and females prefer these males (i.e. find them attractive).

Although Kokko's analysis [61] is a major contribution to our understanding of the relationships between attractiveness and effort, it has some limitations in terms of the interactions that we have identified as being required for a fully consistent model. In particular, the interaction between the male and the female is not considered, so the model cannot predict how the parental investment of a female depends on the attractiveness of her mate.

Phenotypic models such as that of Kokko [61] can be used to analyse direct benefits, but can run into problems when indirect benefits are present. Fisher [62] suggested a self-consistent account of the attractiveness of males when they provide only genetic benefits. These genetic effects can be handled by phenotypic models provided that intergenerational effects are taken into account [63-65]. We need to extend these models to look at care decisions and, hence, incorporate both direct and indirect benefits in a consistent way. 
(e.g. [52,53]). More theoretical and empirical work on the issue of common interests is needed.

- Mating systems and parental care. There is feedback between mating systems and the form of parental care [54], and we need more investigations of how this feedback constrains possible evolutionary outcomes.

- Attractiveness. We need general consistent models of attractiveness in the context of care, and models of the differential allocation hypothesis in particular.

- Multi-dimensional care. In many species, there is a division of labour, with the female specializing in one aspect of care and the male in another. This issue requires more attention from theoreticians, because most models adopt a uni-dimensional approach to parental care, assuming that a single quantity, such as feeding rate, suffices to characterize parental behaviour.

\section{Conclusions}

Parental care research has come a long way since Robert Trivers' seminal dictum 'What governs the operation of sexual selection is the relative parental investment of the sexes in their offspring', [4] but it has still a long way to go. Recent research reveals fascinating new ideas about the conflicts between parents over care, and proposes that care and sexual selection are more intricately related than was previously thought (e.g. [55]). In addition, theoretical models have revealed that the behavioural interactions between the male and the female parents have a major impact on the solution of their conflict over care. However, empirical studies are needed to test the assumptions and predictions of these models. Given that most studies are of birds, further studies are needed to investigate parental care in other organisms, including insects, fishes and mammals. Intriguing theoretical models propose that parents might strategically handicap themselves to manipulate their partner to provide more care. These predictions have so far not been tested and it is not yet clear whether the manipulations are effective in nature.

Parental care is an excellent model paradigm with which to investigate cooperation and conflict between male and female animals (including humans). In our view, further progress depends on a better understanding of how parents interact with each other and with other individuals in the population; that is, with their social environment. The effects of the social milieu and ecology (such as resources and predators) on parental behaviour might be more complicated than we currently acknowledge. Understanding the various effects and interactions is likely to be a hard, although worthwhile, exercise.

\section{Acknowledgements}

We thank I. Cuthill, H. Kokko, P. T. Smiseth, S. Verhulst and three anonymous referees for comments on the article. Our work on parental care has been supported by NERC (GR3/10957) and is now supported by BBSRC (BBS/B/05788). T.S. was also supported by The Royal Society (15056), and Hungarian Science Fund (OTKA T-43390).

\section{References}

1 Clutton-Brock, T.H. (1991) The Evolution of Parental Care, Princeton University Press

2 Balshine-Earn, S. et al. (2002) Conflict and co-operation in parental care. Philos. Trans. R. Soc. Lond. Ser. B 357, 237-404
3 Magrath, M.J.L. and Komdeur, J. (2003) Is male care compromised by additional mating opportunity? Trends Ecol. Evol. 18, 424-430

4 Trivers, R.L. (1972) Parental investment and sexual selection. In Sexual Selection and the Descent of Man (Campbell, B., ed.), pp. 136-179, Aldine

5 Parker, G.A. et al. (2002) Intrafamilial conflict and parental investment: a synthesis. Philos. Trans. R. Soc. Lond. Ser. B 357, 295-307

6 Lessells, C.M. (1999) Sexual conflict in animals. In Levels of Selection in Evolution (Keller, L., ed.), pp. 75-99, Princeton University Press

7 Chapman, T. et al. (2003) Sexual conflict. Trends Ecol. Evol. 18, 41-47

8 Westneat, D.F. and Stewart, I.R.K. (2003) Extra-pair paternity in birds: causes, correlates, and conflict. Annu. Rev. Ecol. Syst. 34, 365-396

9 Grafen, A. and Sibly, R.M. (1978) A model of mate desertion. Anim. Behav. 26, 645-652

10 Yamamura, N. and Tsuji, N. (1993) Parental care as a game. J. Evol. Biol. 6, 103-127

11 Balshine-Earn, S. and Earn, D.J.D. (1997) An evolutionary model of parental care in St. Peter's fish. J. Theor. Biol. 184, 423-431

12 McNamara, J.M. et al. (2000) A dynamic game-theoretic model of parental care. J. Theor. Biol. 205, 605-623

13 Houston, A.I. and McNamara, J.M. (2002) A self-consistent approach to paternity and parental effort. Philos. Trans. R. Soc. Lond. Ser. B $357,351-362$

14 Maynard Smith, J. (1977) Parental investment: a prospective analysis. Anim. Behav. 25, 1-9

15 Webb, J.N. et al. (1999) Multiple patterns of parental care. Anim. Behav. 58, 983-993

16 Kokko, H. (1999) Cuckoldry and the stability of biparental care. Ecol. Lett. 2, 247-255

17 Alonzo, S.H. and Warner, R.R. (2000) Dynamic games and field experiments examining intra- and intersexual conflict: explaining counterintuitive mating behavior in a Mediterranean wrasse, Symphodus ocellatus. Behav. Ecol. 11, 56-70

18 Petrie, M. and Kempenaers, B. (1998) Extra-pair paternity in birds: explaining variation between species and populations. Trends Ecol. Evol. 13, 52-58

19 Griffith, S.C. (2002) Extra-pair paternity in birds: a review of interspecific variation and adaptive function. Mol. Ecol. 11, 2195-2212

20 Grafen, A. (1980) Opportunity cost, benefit and degree of relatedness. Anim. Behav. 28, 967-968

21 Werren, J.H. et al. (1980) Paternity and the evolution of male parental care. J. Theor. Biol. 82, 619-631

22 Queller, D.C. (1997) Why do females care more than males? Proc. $R$. Soc. Lond. Ser. B 264, 1555-1557

23 Wright, J. and Cuthill, I.C. (1989) Manipulation of sex differences in parental care. Behav. Ecol. Sociobiol. 25, 171-181

24 Sanz, J.J. et al. (2000) Differential response by males and females to manipulation of partner contribution in the great tit (Parus major). J. Anim. Ecol. 69, 74-84

25 Smiseth, P.T. and Moore, A.J. (2004) Behavioural dynamics between caring males and females in a beetle with facultative biparental care. Behav. Ecol. 15, 621-628

26 McNamara, J.M. et al. (2002) Do parents make independent decisions about desertion? Anim. Behav. 64, 147-149

27 Dawkins, R. and Carlise, T.R. (1976) Parental investment, mate desertion and a fallacy. Nature 262, 131-133

28 Houston, A.I. and McNamara, J.M. (1999) Models of Adaptive Behaviour, Cambridge University Press

29 McNamara, J.M. et al. (2003) Should young ever be better off with one parent than with two? Behav. Ecol. 14, 301-310

30 McNamara, J.M. et al. (1999) Incorporating rules for responding into evolutionary games. Nature 401, 368-371

31 Schwagmeyer, P.L. and Mock, D.W. (2003) How consistently are good parents good parents? Repeatability of parental care in the house sparrow, Passer domesticus. Ethology 109, 303-313

32 Whittingham, L.A. et al. (1994) Female response to reduced male parental care in birds: an experiment in tree swallows. Ethology 96, 260-269

33 Slagsvold, T. and Lifjeld, J.T. (1988) Ultimate adjustment of clutch size to parental feeding capacity in a passerine bird. Ecology 69, 1918-1922

34 Markman, S. et al. (1995) Male parental care in the orange-tufted sunbird: behavioural adjustments in provisioning and nest guarding effort. Anim. Behav. 50, 655-669 
35 Houston, A.I. and Davies, N.B. (1985) The evolution of cooperation and life history in the dunnock Prunella modularis. In Behavioural Ecology (Sibly, R.M. and Smith, R.H., eds), pp. 471-487, Blackwell Scientific Publications

36 Schwagmeyer, P.L. et al. (2002) Biparental care in house sparrows: negotiation or sealed bid? Behav. Ecol. 13, 713-721

37 Barta, Z. et al. (2002) Sexual conflict about parental care: the role of reserves. Am. Nat. 159, 687-705

38 Smith, H.G. and Härdling, R. (2000) Clutch size evolution under sexual conflict enhances the stability of mating systems. Proc. $R$. Soc. Lond. Ser. B 267, 2163-2170

39 Komdeur, J. et al. (2002) Paternal care and male mate-attraction effort in the European starling is adjusted to clutch size. Proc. R. Soc. Lond. Ser. B 269, 1253-1261

40 Hemborg, C. (1999) Sexual differences in moult-breeding overlap and female reproductive costs in pied flycatchers, Ficedula hypoleuca. J. Anim. Ecol. 68, 429-436

41 Heywood, J.S. (1989) Sexual selection by the handicap mechanism. Evolution 43, 1387-1397

42 Hoelzer, G.A. (1989) The good parent process of sexual selection. Anim. Behav. 38, 1067-1078

43 Soler, J.J. et al. (2001) Nest size affects clutch size and the start of incubation in magpies: an experimental study. Behav. Ecol. 12, 301-307

44 Linville, S.U. et al. (1998) Plumage brightness as an indicator of parental care in northern cardinals. Anim. Behav. 55, 119-127

45 Buchanan, K.L. and Catchpole, C.K. (2000) Song as an indicator of male parental effort in the sedge warbler. Proc. R. Soc. Lond. Ser. B 267, 321-326

46 Smiseth, P.T. and Amundsen, T. (2000) Does female plumage coloration signal parental quality? A male removal experiment with the bluethroat (Luscinia s. svecica). Behav. Ecol. Sociobiol. 47, 205-212

47 Smiseth, P.T. et al. (2001) Is male plumage reflectance correlated with paternal care in bluethroats? Behav. Ecol. 12, 164-170

48 Burley, N. (1988) The differential allocation hypothesis - an experimental test. Am. Nat. 132, 611-628

49 Qvarnström, A. (1997) Experimentally increased badge size increases male competition and reduces male parental care in the collared flycatcher. Proc. R. Soc. Lond. Ser. B 264, 1225-1231
50 Sheldon, B.C. (2000) Differential allocation: tests, mechanisms and implications. Trends Ecol. Evol. 15, 397-402

51 Parker, G.A. (1985) Models of parent-offspring conflict. V. Effects of the behaviour of the two parents. Anim. Behav. 33, 519-533

52 Pen, I. and Weissing, F.J. (2000) Towards a unified theory of cooperative breeding: the role of ecology and life history re-examined. Proc. R. Soc. Lond. Ser. B 267, 2411-2418

53 Härdling, R. et al. (2003) Dynamics of the caring family. Am. Nat. 161, 395-412

54 Székely, T. et al. (2000) Mating patterns, sexual selection and parental care: an integrative approach. In Vertebrate Mating Systems (Apollonio, M. et al., eds), pp. 194-223, World Science Press

55 Kokko, H. and Johnstone, R.A. (2002) Why is mutual mate choice not the norm? Operational sex ratios, sex roles and the evolution of sexually dimorphic and monomorphic signaling. Philos. Trans. R. Soc. Lond. Ser. B 357, 319-330

56 Hammerstein, P. (1996) Darwinian adaptation, population genetics and the streetcar theory of evolution. J. Math. Biol. 34, 511-532

57 Weissing, F.J. (1996) Genetic versus phenotypic models of selection: can genetics be neglected in a long-term perspective? J. Math. Biol. 34, $533-555$

58 Maynard Smith, J. (1982) Evolution and the Theory of Games, Cambridge University Press

59 Eshel, I. (1983) Evolutionary and continuous stability. J. Theor. Biol. 103, 99-111

60 Andersson, M. (1994) Sexual Selection, Princeton University Press

61 Kokko, H. (1998) Should advertising parental care be honest? Proc. $R$. Soc. Lond. Ser. B 265, 1871-1878

62 Fisher, R.A. (1930) The Genetical Theory of Natural Selection, Oxford University Press

63 Pen, I. and Weissing, F.J. (2000) Sexual selection and the sex ratio: an ESS analysis. Selection 1, 59-69

64 Kokko, H. et al. (2002) The sexual selection continuum. Proc. R. Soc. Lond. Ser. B 269, 1331-1340

65 McNamara, J.M. et al. (2003) Quantifying male attractiveness. Proc. R. Soc. Lond. Ser. B 270, 1925-1932

\section{Elsevier.com - Dynamic New Site Links Scientists to New Research \& Thinking}

Elsevier.com has had a makeover, inside and out. Designed for scientists' information needs, the new site, launched in January, is powered by the latest technology with customer-focused navigation and an intuitive architecture for an improved user experience and greater productivity.

Elsevier.com's easy-to-use navigational tools and structure connect scientists with vital information - all from one entry point. Users can perform rapid and precise searches with our advanced search functionality, using the FAST technology of Scirus.com, the free science search engine. For example, users can define their searches by any number of criteria to pinpoint information and resources. Search by a specific author or editor, book publication date, subject area - life sciences, health sciences, physical sciences and social sciences - or by product type. Elsevier's portfolio includes more than 1800 Elsevier journals, 2200 new books per year, and a range of innovative electronic products. In addition, tailored content for authors, editors and librarians provides up-to-the-minute news, updates on functionality and new products, e-alerts and services, as well as relevant events.

Elsevier is proud to be a partner with the scientific and medical community. Find out more about who we are in the About section: our mission and values and how we support the STM community worldwide through partnerships with libraries and other publishers, and grant awards from The Elsevier Foundation.

As a world-leading publisher of scientific, technical and health information, Elsevier is dedicated to linking researchers and professionals to the best thinking in their fields. We offer the widest and deepest coverage in a range of media types to enhance cross-pollination of information, breakthroughs in research and discovery, and the sharing and preservation of knowledge. Visit us at Elsevier.com.

Elsevier. Building Insights. Breaking Boundaries. 